\title{
Drug-induced hallucination: A case/non case study in the French Pharmacovigilance Database
}

\author{
Malak Abou Taam* \\ Paul de Boissieu* \\ Rola Abou Taam** \\ Alexandre Breton* \\ Thierry Trenque*
}

${ }^{*}$ Regional Center for Pharmacovigilance and Pharmacoepidemiology, Reims University Hospitals, Reims

** Department Pediatrics, Pulmonology and Allergy Service, Necker-Enfants Malades Hospital, Paris

FRANCE

\begin{abstract}
Background and Objectives: Hallucinations are sensory perceptions which occur without external stimuli. There are associated with psychiatric disease but also can be related to organic disease and drug or toxic exposure. The purpose of our study was to investigate the association between exposure to medications and the reporting of hallucinations using data from the spontaneous-reporting French Pharmacovigilance Database (FPVD).

Methods: We used the case/noncase method in the FPVD. Cases were all the observations of hallucination with the LLT term "perception disturbances", registered into the FPVD from January 1985 to Jan 2013. Data were expressed as odds ratio (OR) with their 95\% confidence intervals.

Results: Among the 469,181 reports of adverse effects recorded between 1985 and $2013,4,086$ are hallucinations. For about $50 \%$ of these hallucinations were experimented by patient older than 65 years old.

A statistically significant OR was found with several medications included rasagiline (OR 17.6 [95\%CI 10.4-29.8]), zolpidem (OR 12.9 [95\%CI 11.3-14.8]), methylphenidate (OR 9.3 [95\%CI 5.9-14.6]) and baclofene (OR 5.4 [95\%CI 3.7-7.8]). An increased risk of hallucinations was also observed with non central nervous system drugs, including ertapenem (OR 24.0 [95\%CI 14.2-40.5]), voriconazole (OR 12.9[95\%CI 10.2-16.5]) and valacyclovir (OR 9.1 [95\%CI 6.9-11.9]).

Conclusions: This pharmacoepidemiological study describes an association between drugs and hallucinations. This relationship involves not only some already suspected drugs but also other drugs less known to induce such an adverse reaction. Despite the mandatory limits of this kind of study, these data should lead to special precautions in patient at risk.
\end{abstract}




\section{Introduction}

In 1837 the French alienist Esquirol defined hallucinations as follows. "A person is said to labor under a hallucination, or to be a visionary, who has a thorough conviction of the perception of a sensation, when no external object, suited to excite this sensation, has impressed the senses"1,2.

Hallucination is now defined by the Diagnostic and Statistical Manual of Mental Disorders DSMV as “" a sensory perception that has the compelling sense of reality of a true perception but that occurs without external stimulation of the relevant sensory organ" ${ }^{3}$.

It is a relatively common symptom, with a prevalence of 4 to $38 \%{ }^{4}$. The percentage of "normal" subjects reporting hallucinatory experiences is substantial ${ }^{5,6}$. They may be symptomatic of cerebral disease, functional psychoses and can also be induced or worsened by drugs ${ }^{7}$.

In this context, the aim of this study was to investigate the association between exposure to medications and hallucinations.

\section{Methods}

The study used data from the French Pharmacovigilance Database (FPVD) of all adverse reactions occurring with commercially approved drugs in France. This database was established in $1985^{8}$ to register all adverse drug reactions (ADR) reported by health professionals or patients to the French Pharmacovigilance System. Reports are reviewed and analyzed by medically qualified personnel before being entered into the FPVD.

ADR were coded according to the Medical Dictionary for Regulatory Activities (MedDRA $\left.^{\circledR}\right)^{9}$.
Observations reported with hallucination from 1985 to 1 Jan 2013 were reviewed.

The case/non-case method was used to measure disproportionality of the combination between a drug and a particular ADR in the FPVD. Cases were defined as reports corresponding to hallucination from the LLT terms "perception disturbances", and noncases as all other reported ADRs for the same period. Data in the FPVD are anonymous. The odds ratio (OR) is the ratio of reporting of one specific event versus all other events for a given drug compound ${ }^{10}$. The $95 \%$ confidence intervals (CI) were calculated using the Woolf method ${ }^{11}$.

\section{Results}

469,181 reports of ADRs had been recorded in the FPVD from 1985 to 1 January 2013. 4086 patients experimented hallucinations. The average age was 59.8 years $( \pm 28.4) .53 \%$ were older than 65 years old and $9 \%$ were children of less than 15 years. The sex ratio (female/male) was 1.3. ADR are serious in 1691 cases $(41 \%)$.

Underlying disease that can potentially induce hallucination is specified in 592 cases (14\%): 510 patients $(12 \%)$ had psychiatric history, 76 history of dementia and 6 history of Lewis body dementia. Hallucination is associated with other adverse event known to induce this symptom in 167 cases (4\%): 80 drug interactions, 35 withdrawal medication (opioid, benzodiazepine, antidepressant, neuroleptic and baclofene), 19 hyponatraemia (proton pump inhibitors, antidepressant, desmopressin and diuretic), 15 encephalopathy, 9 cerebellous syndrome, 7 anticholinergic syndrome and 2 hypoglycemia.

The type of hallucination is mainly unspecified (69\%), visual (24\%) or auditory 
$(6 \%)$. Patients recovered in $83 \%$. Two patients died after responding inappropriately to hallucinations (one fall and one suicide). For the remaining cases, the outcome is unknown $(7 \%)$, the patient is not yet restored $(8 \%)$ or is recovering (about 2\%).

Overall, a total of 4231 reported medications are suspected, a single one in 3298 cases $(81 \%)$ and more than one in $19 \%$. The onset delay of ADR was less than one month after initiation of the medication in $22 \%$ and in less than two days in $8 \%$. In 5 cases, the ADR was immediate including 3 hallucinations after diazepam injection. Administration was mainly oral $(82 \%)$.
The most important occurrences are zolpidem $(n=250)$, tramadol $(n=240)$, levodopa $(\mathrm{n}=212)$, morphine $(\mathrm{n}=143)$, ofloxacine ( $\mathrm{n}$ =120), trihexyphenidyle $(\mathrm{n}=107)$, ciprofloxacin $(\mathrm{n}=105)$, piribedil $(\mathrm{n}=81)$, oxybutynine $(\mathrm{n}=86)$, omeprazole $(\mathrm{n}=76)$ and voriconazole $(n=74)$.

The highest significant OR is for ertapenem, lisuride, entacapone, selegiline and trihexyphenidyle (Table 1). In the top ten, we find two medications used in non-neurological or psychiatrical indications: ertapenem and oxybutynine.

Parkinson disease therapy was mainly implicated with 408 cases (Table 2a). Benzodi-

Table 1

Top ten of statistically significant OR for the association between medication and hallucinations in the FPVD*.

Drug OR (95\% CI)

\begin{tabular}{ll}
\hline Ertapenem & $24.0(14.2-40.5)$ \\
\hline Lisuride & $23.1(13.5-39.6)$ \\
\hline Entacapone & $20.4(12.8-32.4)$ \\
\hline Selegiline & $19.2(12.9-28.7)$ \\
\hline Trihexyphenidyle & $17.8(14.5-21.9)$ \\
\hline Scopolamine & $17.7(11.9-26.2)$ \\
\hline Pramipexole & $17.7(11.8-26.5)$ \\
\hline Rasagiline & $17.6(10.4-29.8)$ \\
\hline Levodopa + carbidopa & $17.2(12.9-22.8)$ \\
\hline Oxybutynine & $16.6(13.2-20.8)$ \\
\hline
\end{tabular}

* 95\% CI calculated using Woolf's method, OR = odd ratio, CI = confidence interval, FPVD = French Pharmacovigilance Database.

azepines and benzodiazepine-like therapies were also well-represented $(\mathrm{n}=365)$ including 250 cases with zolpidem (Table 2 b). Opioids were involved in many cases and mainly tramadol $(n=240)$ (Table 2c). Other anticholinergic medications induced hallucinations as antidepressants (duloxetine...), anxiolytics (hydroxyzine...), neuroleptic (aripiprazole...), antihistaminics therapies (promethazine, mequitazine), overbladder therapies (oxybutynine, trospium), treatment of the neuroleptic-induced parkinsonism (trihexyphenidyle) and analgesic (nefopam) (Table 2d, 2e). Other drugs with nervous central system activities produced also hallucination with significant OR including antiepileptic (gabapentine, 
Table 2

CNS medications with statistically significant OR for the association with hallucination in the FPVD*.

\begin{tabular}{|c|c|c|c|c|}
\hline Drug & Hallucination & All reports FPVD & OR & $(95 \% \mathrm{CI})$ \\
\hline \multicolumn{5}{|l|}{ a-Parkinson disease therapy } \\
\hline Bromocriptine & 91 & 902 & 13.0 & $(10.5-16.2)$ \\
\hline Entacapone & 21 & 139 & 20.4 & $(12.8-32.4)$ \\
\hline Levodopa + carbidopa & 56 & 432 & 17.2 & $(12.9-22.8)$ \\
\hline Levodopa + benserazide & 138 & 1171 & 15.7 & $(13.1-18.8)$ \\
\hline Levodopa, entacapone, carbidopa & 18 & 149 & 15.7 & $(9.6-25.7)$ \\
\hline Lisuride & 16 & 95 & 23.1 & $(13.5-39.6)$ \\
\hline Pergolide & 16 & 192 & 10.4 & $(6.2-17.3)$ \\
\hline Piribedil & 81 & 699 & 15.2 & $(12.0-19.2)$ \\
\hline Pramipexole & 27 & 202 & 17.7 & $(11.8-26.5)$ \\
\hline Rasagiline & 16 & 120 & 17.6 & $(10.4-29.8)$ \\
\hline Selegiline & 28 & 195 & 19.2 & $(12.9-28.7)$ \\
\hline Trihexyphenidyle & 107 & 808 & 17.8 & $(14.5-21.9)$ \\
\hline Tropatepine & 29 & 596 & 5.9 & $(4.0-8.5)$ \\
\hline \multicolumn{5}{|c|}{ b-Benzodiazepine and benzodiazepine-like therapies } \\
\hline Bromazepam & 37 & 2863 & 1.5 & $(1.1-2.1)$ \\
\hline Clonazepam & 37 & 1907 & 2.3 & $(1.6-3.1)$ \\
\hline Lorazepam & 34 & 1680 & 2.4 & $(1.7-3.3)$ \\
\hline Oxazepam & 26 & 1418 & 2.1 & $(1.4-3.1)$ \\
\hline Triazolam & 12 & 370 & 3.8 & $(2.1-6.8)$ \\
\hline Zolpidem & 250 & 2586 & 12.9 & $(11.3-14.8)$ \\
\hline Zopiclone & 74 & 1942 & 4.6 & $(3.6-5.8)$ \\
\hline \multicolumn{5}{|l|}{ c-Opioids therapies } \\
\hline Buprenorphine & 38 & 1946 & 2.3 & $(1.7-3.1)$ \\
\hline Dextromethorphane & 11 & 276 & 4.7 & $(2.6-8.7)$ \\
\hline Morphine & 143 & 3939 & 4.4 & $(3.7-5.2)$ \\
\hline Nalbuphine & 15 & 301 & 6.0 & $(3.6-10.1)$ \\
\hline Opium & 14 & 604 & 2.7 & $(1.6-4.6)$ \\
\hline Oxycodone & 21 & 334 & 7.7 & $(4.9-11.9)$ \\
\hline Tramadol & 240 & 4784 & 6.3 & $(5.5-7.2)$ \\
\hline \multicolumn{5}{|c|}{ d-Antidepressants, anxiolytics and neuroleptics } \\
\hline Agomelatine & 7 & 227 & 3.6 & $(1.7-7.7)$ \\
\hline Amitriptyline & 52 & 1864 & 3.3 & $(2.5-4.3)$ \\
\hline Aripiprazole & 10 & 502 & 2.3 & $(1.2-4.3)$ \\
\hline Citalopram & 26 & 1438 & 2.1 & $(1.4-3.1)$ \\
\hline Clomipramine & 46 & 2256 & 2.4 & $(1.8-3.2)$ \\
\hline
\end{tabular}

*95\% CI calculated using Woolf's method, OR = odd ratio, CI = confidence interval, FPVD = French Pharmacovigilance Database. 
Table 2

CNS medications with statistically significant OR for the association with hallucination in the FPVD* (continuation).

\begin{tabular}{|c|c|c|c|c|}
\hline \multirow{2}{*}{$\frac{\text { Drug }}{\text { d-Antidepressants, anxiolytics }}$} & \multirow[t]{2}{*}{ Hallucination } & \multirow[t]{2}{*}{ All reports FPVD } & \multicolumn{2}{|c|}{ OR $(95 \% \mathrm{CI})$} \\
\hline & & & & \\
\hline Duloxetine & 21 & 496 & 5.1 & $(3.3-7.8)$ \\
\hline Fluvoxamine & 17 & 749 & 2.6 & $(1.6-4.3)$ \\
\hline Haloperidol & 30 & 2139 & 1.6 & $(1.1-2.3)$ \\
\hline Hydroxyzine & 45 & 1828 & 2.9 & $(2.1-3.9)$ \\
\hline Paroxetine & 57 & 3405 & 2.0 & $(1.5-2.5)$ \\
\hline Sertraline & 22 & 1116 & 2.3 & $(1.5-3.5)$ \\
\hline Venlafaxine & 28 & 1891 & 1.7 & $(1.2-2.5)$ \\
\hline \multicolumn{5}{|c|}{ e-Other medications with anticholinergic activity } \\
\hline Atropine & 42 & 820 & 6.2 & $(4.5-8.5)$ \\
\hline Butylscopolamine & 3 & 43 & 8.5 & $(2.6-27.6)$ \\
\hline Mequitazine & 18 & 286 & 7.7 & $(4.8-12.4)$ \\
\hline Nefopam & 70 & 981 & 8.9 & $(6.9-11.3)$ \\
\hline Oxybutynine & 86 & 689 & 16.6 & $(13.2-20.8)$ \\
\hline Piperazine & 2 & 39 & 6.2 & $(1.5-25.5)$ \\
\hline Promethazine & 14 & 315 & 5.3 & $(3.1-9.1)$ \\
\hline Pseudoephedrine chlorydrate & 9 & 140 & 7.8 & $(4.0-15.4)$ \\
\hline Scopolamine & 29 & 217 & 17.7 & $(11.9-26.2)$ \\
\hline Trospium chloride & 7 & 108 & 7.9 & $(3.7-17.0)$ \\
\hline \multicolumn{5}{|l|}{ f-Other CNS therapies } \\
\hline Baclofene & 30 & 665 & 5.4 & $(3.7-7.8)$ \\
\hline Buflomedil & 15 & 843 & 2.1 & $(1.2-3.4)$ \\
\hline Clobutinol & 13 & 289 & 5.4 & $(3.1-9.4)$ \\
\hline Donepezil & 18 & 775 & 2.7 & $(1.7-4.3)$ \\
\hline Gabapentine & 22 & 886 & 2.9 & $(1.9-4.4)$ \\
\hline Galantamine & 7 & 328 & 2.5 & $(1.2-5.3)$ \\
\hline Ketamine & 14 & 554 & 3.0 & $(1.7-5.0)$ \\
\hline Leviracetam & 28 & 835 & 4.0 & $(2.7-5.8)$ \\
\hline Memantine & 16 & 442 & 4.3 & $(2.6-7.1)$ \\
\hline Methylphenidate & 20 & 267 & 9.3 & $(5.9-14.6)$ \\
\hline Modafinil & 6 & 123 & 5.8 & $(2.6-13.3)$ \\
\hline Oxetorone & 4 & 129 & 3.6 & $(1.3-9.9)$ \\
\hline Pregabaline & 32 & 1176 & 3.2 & $(2.2-4.6)$ \\
\hline Sodium 4-hydroxybutyrate & 11 & 97 & 14.6 & $(7.8-27.4)$ \\
\hline Topiramate & 17 & 609 & 3.3 & $(2.0-5.3)$ \\
\hline Varenicline & 9 & 521 & 2.0 & $(1.0-3.9)$ \\
\hline
\end{tabular}

*95\% CI calculated using Woolf's method, OR = odd ratio, CI = confidence interval, FPVD = French Pharmacovigilance Database. 
leviracetam, pregabaline and topiramate), Alzheimer therapies (donepezil, galantamine, memantine), migraine treatment (oxetorone), analgesic (ketamine), hyperactivity disorder and/or narcolepsia treatment (methylphenidate, modafinil, sodium-4-hydroxybutyrate), an antispastic treatment (baclofene) and an antitussive therapy (clobutinol) (Table 2f).
An increased risk of hallucinations was also observed with non central nervous system (NCNS) drugs (Table 3), including ertapenem, voriconazole, valacyclovir, oseltamivir, mefloquine, ciprofloxacin and omeprazole. Other drug presented less important OR but statistically significant: tacrolimus and prednisolone.

Table 3

Description of cases with statistically significant odds ratio (ORs) for the association between NCNS medication and hallucination in the FPVD*.

\begin{tabular}{lccccc} 
Drug & $\begin{array}{c}\text { All reports } \\
\text { FPVD }\end{array}$ & $\begin{array}{c}\text { Hallucination } \\
\text { cases }(\mathrm{n})\end{array}$ & $\begin{array}{c}\text { Sex ratio } \\
(\mathrm{F} / \mathrm{H})\end{array}$ & Mean age (y) & OR (95\% CI) \\
\hline Aciclovir & 2045 & 38 & 1.6 & 58.4 & $4.0(2.9-5.6)$ \\
\hline Ciprofloxacin & 3342 & 105 & 0.7 & 66.6 & $3.8(3.1-4.6)$ \\
\hline Efavirenz & 2337 & 35 & 0.35 & 42.2 & $1.7(1.2-2.4)$ \\
\hline Ertapenem & 98 & 17 & 0.7 & 73.8 & $24.0(14.2-40.5)$ \\
\hline Ivabradine & 100 & 3 & 0 & 83 & $3.5(1.1-11.1)$ \\
\hline Levofloxacin & 1610 & 49 & 0.9 & 74.8 & $3.6(2.7-4.8)$ \\
\hline Mefloquine & 801 & 40 & 1.2 & 32.5 & $6.0(4.4-8.3)$ \\
\hline Metoprolol & 408 & 9 & 1.25 & 59.7 & $2.6(1.3-5.0)$ \\
\hline Montelukast & 322 & 9 & 0.8 & 19.2 & $3.2(1.7-6.4)$ \\
\hline Moxifloxacin & 623 & 12 & 1.4 & 72.5 & $2.2(1.3-3.9)$ \\
\hline Norfloxacin & 1593 & 37 & 5.7 & 68.6 & $2.7(1.9-3.8)$ \\
\hline Ofloxacin & 3923 & 120 & 1.2 & 62.9 & $3.7(3.1-4.4)$ \\
\hline Omeprazole & 4423 & 76 & 0.92 & 69.0 & $2.0(1.6-2.5)$ \\
\hline Oseltamivir & 258 & 16 & 1.1 & 30.8 & $7.5(4.5-12.5)$ \\
\hline Pantoprazole & 1284 & 21 & 1.3 & 73.1 & $1.9(1.2-2.9)$ \\
\hline Piperacilline+tazobactam & 793 & 14 & 1.8 & 74.1 & $2.0(1.2-3.5)$ \\
\hline Proguanil & 802 & 13 & 1.2 & 41 & $1.9(1.1-3.2)$ \\
\hline Valacyclovir & 806 & 56 & 1.4 & 76.8 & $9.1(6.9-11.9)$ \\
\hline Voriconazole & 735 & 74 & 0.48 & 56.2 & $12.9(10.2-16.5)$ \\
\hline
\end{tabular}

* 95\% CI calculated using Woolf's method, OR = odd ratio, CI = confidence interval, NCNS = non central nervous system, FPVD = French Pharmacovigilance Database. 


\section{Discussion}

It is sometimes difficult to establish if hallucination is caused by a drug or by an underlying illness. Indeed, hallucinations are also frequent in schizophrenia and related psychotic disorders.

Our study shows that there is an increased risk of reporting of hallucination, during treatment with several medications. They have sometimes but not always a known nervous central system activity. Several cases of drug-induced hallucination are published but to the best of our knowledge, this is the first review of drug-induced hallucination based on a large pharmacoepidemiological study.

Hallucinations will normally resolve after withdrawal of the causative drug.

The elderly, and those with a previous history of psychiatric illness, are at increased risk of drug-induced hallucination. Hallucinations caused by drugs are commonly visual but in our study the type is mainly unspecified in the coding.

Several differential diagnoses have to be taken into account: in more severe cases of syndrome of inappropriate secretion of antidiuretic hormone or hyponatraemia, anticholinergic syndrome, serotonin syndrome ${ }^{12}$ as auditory and visual hallucinations are included in Sternbach's diagnostic criteria. Hallucinations can also appear after SRI discontinua$\operatorname{tion}^{13}$. However, some types of visual hallucination in Charles Bonnet syndrome (CBS) have been treated with SRI ${ }^{14}$ and CBS is a condition characterized by visual hallucinations in cognitively normal patients with acquired vision loss. Drug-induced hallucinations in healthy elderly subjects should also be investigated as possible predictive marker of Lewy body disease (DLB). It is then very important to have a sufficient accuracy in the data when analyzing those observations and more than all when treating those patients.

Zolpidem is the more frequent medication suspected in hallucination but fluoroquinolones represent also an important class. Neuropsychiatric symptoms are common also in Parkinson's disease (PD) but the other common cause is the use of dopaminergic drugs. Aggravation of psychosis with hallucinations is described also with neuroleptic drugs maybe because of their antimuscarinic properties.

Actually, drug-induced hallucinations most often reflect anticholinergic properties. There are several anticholinergic drugs, some are structurally related to atropine. Their ability to cause vivid and sometimes exotic hallucinations is well-known and has led to their misuse. Nefopam is a centrally acting nonopioid analgesic. It should be used with caution in the elderly and in conjunction with other drugs that have anticholinergic activity. Antimuscarinic therapy for overactive blad$\operatorname{der}^{15}$ as oxybutynin like other drugs used in urology produces anticholinergic symptoms and overdoses causes hallucinations. Solifenacin, which is a more recent, can induce also hallucination but only one case has been reported in our study ${ }^{15}$. First generation H1antihistamines have prominent anticholinergic actions which may contribute to the CNS stimulation seen in some patients. Overdose of various classic H1 antagonists was repeatedly reported to result in toxic psychoses with hallucinations. With "second generation"/piperidine drugs, they do also occur. Directs central nervous system actions of tricyclic antidepressant cause hallucinations. Antimuscarinic effects are especially pronounced with amitriptyline and less with imipramine and clomipramine. Concerning serotonin uptake inhibitors, hallucination can be the result of its anticholinergic properties but also in case of serotoninergic syndrome. Monoamine oxidase inhibitors overdose causes hallucinations for the same reason. 
The neurochemical changes occurring in PD and DLB strongly support a central role of cholinergic and serotonergic imbalance (cholinergic hypoactivity and serotonergic hyperactivity), both in cortical visual areas and in the brainstem, for the genesis of visual hallucination. In addition, Lauterbach suggested that SRI drugs may induce psychotic symptoms by serotonin receptor (5-HT-3) mediated dopamine release in the ventral striate ${ }^{16}$.

Benzodiazepine can produce visual hallucinations. Elderly patients are especially at risk and should take lower-than-usual dose. Baclofen is known in case of abrupt discontinuation to produce withdrawal symptoms including hallucinations. It inhibits GABAmediated spinal reflexes and is variously postulated to inhibit dopaminergic activity or to enhance it by amphetamine-like effect.

Ketamine is currently used as an antihyperalgesic and opioid-sparing agent during the perioperative period. It can also be use as recreational use.

Hallucinations under antiepileptic are described and also in case of withdrawal ${ }^{17}$ : valproic, zonisamide ${ }^{18}$, carbamazepine ${ }^{19}$, levetiracetam $^{20}$, topiramate and anti-N-MethylD-Aspartate receptor encephalitis ${ }^{21}$.

Opioid agonists are marketed as strong analgesics (fentanyl, morphine and methadone), mild-to-moderate analgesics (codeine...), antitussives (codeine, dextrometorphan), antidiarrheals (loperamide), anesthesia adjuncts (fentanyl, sulfentanyl)... Their psycomimetic effects include hallucinations. Tramadol has mu-agonist properties and also inhibits reuptake of norepinephrine and serotonine which can explain his higher ROR.

Methylphenidate is a piperidine derivative structurally related to amphetamine. It is marketed for narcolepsy and attention deficit disorder. Hallucination is then a well-known and important identified risk of methylphenidate.
Concerning beta-adrenergic blocker, it is claimed that lipophilic agents, such as propranolol and metoprolol can cross the blood-brain barrier, whereas hydrophilic beta-blockers cannot. The mechanism of metoprolol-induced delirium is not fully understood. It could be due to impairment of hepatic metabolism (especially in the ageing liver) and complex neurotransmitter-related effects on brain beta-adrenoceptors and serotonin (5-HT) receptors ${ }^{22}$. According Middlemiss, propranolol also possesses serotonin-antagonist properties ${ }^{23}$.

Glucocorticosteroids cause a variety of behavioral abnormalities including schizophrenic-type psychosis. Tacrolimus is usually given with corticosteroids and so the specificity of hallucination is difficult to define.

Hanneken hypothesized that proton pump inhibitors (PPI) may disrupt normal horizontal cell-photoreceptor cell feedback interactions and thereby alter spatial and chromatic perception. They demonstrated that PPI with the core structure 2-pyridyl-methylsulfinyl benzimidazole can induce or enhance visual hallucinations in wet macular degeneration patients with a breakdown of the outer blood-retinal barrier ${ }^{24}$. Another explanation for those hallucinations is the hyponatraemia which can be induced by PPI.

Montelukast is a leukotriene receptor antagonist used in treatment of asthma and has been described to induce hallucination ${ }^{25,26}$.

Although adverse drug reactions are a well-recognized cause of mental status changes in the elderly, antimicrobials are not often implicated.

Visual hallucinations are rare among patients who are only taking fluoquinolones but are relatively frequent among patients using multiple medications. Visual and other sensory symptoms are usually dose related and transient. Fluoroquinolones dose regimens should be reduced in cases of renal 
failure. In an experimental study, Segev determined that fluoquinolones displace the Gamma-aminobutyric acid (GABA) from its receptor, resulting in the general stimulation of the central nervous system, which is the basis for this adverse effect ${ }^{27,28}$. GABA receptor antagonism of fluoroquinolone can maybe explain their neurological properties including the hallucination. Fluoroquinolone should be avoided in patient with psychotic disorder. Only few cases of levofloxacin induced hallucinations have been described in literature ${ }^{29}$. Our 49 cases allow us to specify the profile of patients.

Other antibiotics can be involved as ertapenem which is a carbapenem antibiotic with a high lipophilicity, a central nervous penetration, and a great volume of distribution. Because both clarithromycin and tramadol are metabolised by CYP3A4 in the liver, symptoms were worsened by the drug-drug interaction ${ }^{30}$.

Concerning antifungal azoles ${ }^{31}$, systematic therapeutic drug monitoring of voriconazole is mandatory for some authors because of the risk of CYP polymorphism resulting sometimes in hallucination $^{32}$. Multiple regression analysis of voriconazole concentration according to Dolton and et al. found associations between increasing patient age and coadministration of proton pump inhibitors and increased concentrations ${ }^{33}$.

When prescribing antimalarial agents ${ }^{34}$, neuropsychiatric reactions occur even during a prophylactic use of mefloquine and are well-described.

Neurotoxicity secondary to acyclovir is associated with high plasma concentrations. Valaciclovir is the L-valyl ester of acyclovir that is rapidly metabolized to yield acyclovir and valine. Hallucination is especially likely in the presence of renal insufficiency.

Efavirenz, another antiviral used in $\mathrm{Hu}-$ man Immunodeficiency Virus has according
Gatch a LSD-like activity mediated via the 5HT(2A) receptor ${ }^{35}$.

The mechanisms of drug-induced hallucinations are poorly understood and probably numerous. Anyway 5-HT2A receptors seem to be clearly involved ${ }^{36,37}$.

We can hypothesize that interaction or renal failure can induce overdosage and expose elderly patients to conditions which result in hallucination as hyponatraemia, encephalopathy and serotonin syndrome.

Some limitations of this retrospective study deserve to be mentioned. There are several potential pitfalls inherent to the use of disproportionality measures in spontaneous reporting databases to estimate risk. For example, selection bias due to spontaneous reporting ${ }^{38}$ (such as underreporting and notoriety bias). However, as stated by Mannesse $e t$ $a l .{ }^{39}$, non-selective underreporting and overreporting does not have any significant influence on the ROR estimation, since it affects both the numerator and the denominator.

The inaccuracy of several observations cannot lead us to exclude other aetiologies as toxicomania when we know for instance that the scores on a hallucination proneness correlated positively to use of cannabis ${ }^{40}$.

The difficulty to distinguish drug effects from those of the underlying disorder is an obvious problem. The temporal relationship and the effect of dechallenge are particularly important in assessing the role of the drug. Anyway, we cannot exclude multifactorial causes, especially in the elderly with polymedication. In those cases, iatrogenic hallucination secondary to medications seems to be preventable. Compliance with safety precautions is more necessary than ever and those drugs should be also avoided in patient with psychotic disorder. 


\section{Conclusions}

There is a long list of compounds that may lead to hallucinations, among which zolpidem is the most common. Early diagnosis of drug-induced hallucination and withdrawal of the offending agent is essential. It can be prevented by avoiding polypharmacy and adhering to the saying 'start low and go slow'. Special care is also needed when prescribing for people with renal impairment or in the elderly and for patient with psychotic disorder.

This study has been presented to draw attention of the psychiatrists and other health professionals to the hazardous effect of medications on mental health and especially with non psychiatric medication.

\section{Acknowledgements}

The authors would like to thank all 31 regional centres of the French Pharmacovigilance System for their assistance.

No sources of funding were used to conduct this study or prepare this manuscript. The authors have no conflicts of interest to declare.

\section{References}

1. Blom JD. A Dictionary of Hallucinations. Springer. 2009; 553 p.

2. Peyroux E, Thibaut F, Franck N. Hallucinations. EMC (Elsevier Masson SAS, Paris), Psychiatrie 37-120-A-10. 2013.

3. Diagnostic and Statistical Manual of Mental Disorders, Fifth Edition. American Psychiatric Association. 2013.

4. Temmingh H, Stein DJ, Seedat S, Williams DR. The prevalence and correlates of hallucinations in a general population sample: Findings from the South African Stress and Health Study. Afr J Psychiatry. 2011; 14(3): 211-217.
5. Tien AY. Distributions of hallucinations in the population. Soc Psychiatry Psychiatr Epidemiol. 1991; 26(6): 287-292.

6. Barrett TR, Etheridge JB. Verbal hallucinations in normals, I: People who hear 'voices'. Applied Cognitive Psychology. 1992; 379-387.

7. Ohayon MM. Prevalence of hallucinations and their pathological associations in the general population. Psychiatry Res. 2000; 97(2-3): 153-164.

8. Moore N, Biour M, Paux G, Loupi E, Begaud B, Boismare $\mathrm{F}$, et al. Adverse drug reaction monitoring: Doing it the French way. Lancet. 1985; 2(8463): 1056-1058.

9. Brown EG, Wood L, Wood S. The medical dictionary for regulatory activities (MedDRA). Drug Saf Int J Med Toxicol Drug Exp. 1999; 20(2): 109-117.

10. Moore N, Kreft-Jais C, Haramburu F, Noblet C, Andrejak M, Ollagnier M, et al. Reports of hypoglycaemia associated with the use of ACE inhibitors and other drugs: a case/non-case study in the French pharmacovigilance system database. Br J Clin Pharmacol. 1997; 44(5): 513-518.

11. Woolf B. On estimating the relation between blood group and disease. Ann Hum Genet. 1955; 19(4): 251-253.

12. Ameen S, Praharaj SK. Functional auditory hallucinations in a case of serotonin syndrome. J Neuropsychiatry Clin Neurosci. 2013; 25(1): E60-61.

13. Yasui-Furukori N, Kaneko S. Hallucination induced by paroxetine discontinuation in patients with major depressive disorders. Psychiatry Clin Neurosci. 2011; 65(4): 384-385.

14. Lang UE, Stogowski D, Schulze D, Domula M, Schmidt E, Gallinat J, et al. Charles Bonnet Syndrome: Successful treatment of visual hallucinations due to vision loss with selective serotonin reuptake inhibitors. J Psychopharmacol. 2007; 21(5): 553-555.

15. Stuhec M. Solifenacin-induced delirium and hallucinations. Gen Hosp Psychiatry. 2013; 35(6): 682. e3-4.

16. Lauterbach EC. Dopaminergic hallucinosis with fluoxetine in Parkinson's disease. Am J Psychiatry. 1993; 150(11): 1750 .

17. De Laat SAA, Hillegers MHJ, Jansen FE, Braun KP, de Graeff-Meeder ER. Hallucinations after withdrawal of valproic acid. Pediatrics. 2012; 130(1): e236-238.

18. Akman CI, Goodkin HP, Rogers DP, Riviello JJ Jr. Visual hallucinations associated with zonisamide. Pharmacotherapy. 2003; 23(1): 93-96.

19. Beitinger PA, Kirmeier T, Bronisch T, Wetter TC. Association of auditory hallucinations and anticonvulsant hypersensitivity syndrome with carbamazepine treatment. A case report. Pharmacopsychiatry. 2006; 39(5): 192-193. 
20. Shakya DR, Dutta A, Gautam R. Hallucination in a seizure patient using levetiracetam: A case report. Case Rep Med. 2012; 2012: 706243.

21. Punja M, Pomerleau AC, Devlin JJ, Morgan BW, Schier JG, Schwartz MD. Anti-N-methyl-D-aspartate receptor (anti-NMDAR) encephalitis: An etiology worth considering in the differential diagnosis of delirium. Clin Toxicol (Phila). 2013; 51(8): 794-797.

22. Fisher AA, Davis M, Jeffery I. Acute delirium induced by metoprolol. Cardiovasc Drugs Ther Spons Int Soc Cardiovasc Pharmacother. 2002; 16(2): 161-165.

23. Middlemiss DN, Hutson PH. The 5-HT1B receptors. Ann N Y Acad Sci. 1990; 600: 132 147; discussion 347-348.

24. Hanneken AM, Babai N, Thoreson WB. Oral proton pump inhibitors disrupt horizontal cell-cone feedback and enhance visual hallucinations in macular degeneration patients. Invest Ophthalmol Vis Sci. 2013; 54(2): 1485-1489.

25. Wallerstedt SM, Brunlöf G, Sundström A, Eriksson AL. Montelukast and psychiatric disorders in children. Pharmacoepidemiol Drug Saf. sept 2009; 18(9): 858-864.

26. Kocyigit A, Gulcan Oksuz B, Yarar F, Uzun F, Igde M, Islek I. Hallucination development with montelukast in a child with asthma: Case presentation. Iran J Allergy Asthma Immunol. 2013; 12(4): 397-399.

27. Segev S, Rehavi M, Rubinstein E. Quinolones, theophylline, and diclofenac interactions with the gammaaminobutyric acid receptor. Antimicrob Agents Chemother. 1988; 32(11): 1624-1626.

28. Sangalli BC. Role of the central histaminergic neuronal system in the CNS toxicity of the first generation H1antagonists. Prog Neurobiol. 1997; 52(2): 145-157.

29. Lertxundi U, Palacios RH, Gutierrez FC, Echaburu SD, García MG, Gómez CA. Levofloxacin-Induced Delirium in a Patient Suffering from Schizoaffective Disorder and Multiple Sclerosis. Curr Drug Saf. 2013.

30. Kovács G, Péter L. [Complex hallucination (visualauditory) during coadministration of tramadol and clarithromycin]. Neuropsychopharmacol Hung. 2010; 12(1): 309-312.

31. Zonios DI, Gea-Banacloche J, Childs R, Bennett JE. Hallucinations during voriconazole therapy. Clin Infect Dis. 2008; 47(1): e7-e10.

32. Lemaitre F, Barbaz M, Scailteux L-M, Uhel F, Tadié $\mathrm{J}-\mathrm{M}$, Verdier M-C, et al. A case-report of unpredictable and massive voriconazole intoxication in a patient with extensive CYP2C19 and CYP2C9 polymorphisms. Drug Metab Pharmacokinet. 2013; 28(5): 439-441.
33. Dolton MJ, Ray JE, Chen SC-A, Ng K, Pont LG, McLachlan AJ. Multicenter study of voriconazole pharmacokinetics and therapeutic drug monitoring. Antimicrob Agents Chemother. 2012; 56(9): 4793-4799.

34. Phillips-Howard PA, ter Kuile FO. CNS adverse events associated with antimalarial agents. Fact or fiction? Drug Saf. 1995; 12(6): 370-383.

35. Gatch MB, Kozlenkov A, Huang R-Q, Yang W, Nguyen JD, González-Maeso J, et al. The HIV antiretroviral drug efavirenz has LSD-like properties. Neuropsychopharmacology. 2013; 38(12): 2373-2384.

36. Baggott MJ, Siegrist JD, Galloway GP, Robertson LC, Coyle JR, Mendelson JE. Investigating the mechanisms of hallucinogen-induced visions using 3,4-methylenedioxyamphetamine (MDA): A randomized controlled trial in humans. PloS One. 2010; 5(12): e14074.

37. Kometer M, Schmidt A, Jäncke L, Vollenweider FX. Activation of serotonin $2 \mathrm{~A}$ receptors underlies the psilocybin-induced effects on oscillations, N170 visual-evoked potentials, and visual hallucinations. J Neurosci Off J Soc Neurosci. 2013; 33(25): 10544-10551.

38. Pariente A, Gregoire F, Fourrier-Reglat A, Haramburu F, Moore N. Impact of safety alerts on measures of disproportionality in spontaneous reporting databases: The notoriety bias. Drug Saf. 2007; 30(10): 891-898.

39. Mannesse CK, van Puijenbroek EP, Jansen PAF, van Marum RJ, Souverein PC, Egberts TCG. Hyponatraemia as an adverse drug reaction of antipsychotic drugs:A casecontrol study in VigiBase. Drug Saf. 2010; 33(7): 569-578.

40. García Montes JM, Zaldívar Basurto F, Moreno Montoya M, Flores Cubos P. Relationship between drug use and psychopathological variables of risk in university students. Psicothema. 2013; 25(4): 433-439.

Corresponding author:

Malak ABOU TAAM, PharmD

Regional Center for Pharmacovigilance and

Pharmacoepidemiology

Reims University Hospitals

Reims

France

Avenue du General Koenig

51092 REIMS, France

Phone: 33.3 .26 .78 .77 .80

Fax: 33.3.26.83.23.79

E-mail: maboutaam@chu-reims.fr 